\title{
Microbiology Specimen Link Identifier
}

National Cancer Institute

\section{Source}

National Cancer Institute. Microbiology Specimen Link Identifier. NCI Thesaurus. Code C162018.

A sequence of characters used as a linkage between related microbiology specimen identifier records. 